to healthy skin[1]. ACKR2 is a scavenging receptor of inflammatory CC chemokines and has been proposed as a regulator of cutaneous inflammation in psoriasis. It has not been studied in PsA.

Objectives: To compare the transcriptome of PsA lesional, PsA uninvolved and healthy control skin and evaluate ACKR2 expression in PsA.

Methods: Biopsies were taken from healthy control $(\mathrm{HC})$ skin and paired lesional and uninvolved skin from patients with PsA. Libraries for bulk RNA sequencing were prepared from polyA selected RNA and sequenced on NovaSeq 6000. Sequencing data were analysed using Searchlight2. ACKR2 mRNA expression was validated by qPCR. RNAscope was used to localise ACKR2 expressing cells and sections were co-stained with podaplanin or stained in serial sections with CD45. Chemokine protein expression in skin was evaluated using Luminex technology.

Results: Nine $\mathrm{HC}$ and 9 paired skin samples from patients with PsA were sequenced. The PsA skin lesions (PsA L) formed a distinct population in the transcriptomic principal component analysis (PCA) plot while HC and PsA uninvolved skin (PsA U) were overlapping. Only 15 genes were differentially expressed between $\mathrm{HC}$ and PSA $U$ and none coded for chemokines. There were however significantly upregulated chemokines and receptors in PsA L. Unexpectely, ACKR2 was the $2^{\text {nd }}$ most upregulated chemokine receptor in PsA $L$ with unchanged expression in PsA U compared with HC (PsA L vs HC log2fold 3.38, p.adj=9.51E-41; PsA L vs PsA U log2fold 3.58, p.adj=3.24E-45; PsA U vs HC log2fold -0.2, p.adj=0.732).

The upregulation of ACKR2 in PSA $L$ and unchanged expression in PsA $U$ was confirmed by qPCR. RNAscope demonstrated strong expression of ACKR2 in the suprabasal layer of the epidermis in PSA L. In HC and PSA U, only occasional ACKR2 positive cells were seen in the epidermis. ACKR2 was expressed in lymphatic vessel walls but was not observed in CD45+ leukocytes.

Provisional skin chemokine protein expression data showed poor correlation between mRNA levels and protein expression for the ACKR2 ligands CCL2, CCL3, CCL7, CCL8, CCL11, CCL13 and CCL22 in HC and PSA U, with negative correlation between ACKR2 mRNA expression and CCL2, CCL8 and CCL11 protein expression. In PsA L, chemokine mRNA correlated with protein expression, but protein expression of chemokine ligands did not correlate with ACKR2 expression.

Conclusion: This data set shows expected upregulation of chemokines and their receptors in PsA $L$ but relatively unchanged gene expression in PsA U, which contrasts to previous studies in psoriasis. Notably, this study demonstrates a strong upregulation of ACKR2 in keratinocytes in PsA L, with unchanged expression in PsA U. The RNA expression and preliminary protein data suggest that ACKR2 has little effect on the levels of its ligands in PsA skin lesions. However, this study may have missed local effects of ACKR2 in the epidermis.

References:

[1] Singh, M.D., et al., Elevated expression of the chemokine-scavenging receptor D6 is associated with impaired lesion development in psoriasis. Am J Pathol, 2012. 181(4): p. 1158-64.

Acknowledgments: Funded by the Chief Scientist Office and a private donation to the University of Glasgow. Dr Sabarinadh Chilaka helped to prepare libraries for RNA sequencing.

Disclosure of Interests: Hanna Johnsson: None declared, John Cole: None declared, Gillian Wilson: None declared, Marieke Pingen: None declared, Fiona McMonagle: None declared, Susan Holmes: None declared, lain Mclnnes Grant/ research support from: Bristol-Myers Squibb, Celgene, Eli Lilly and Company, Janssen, and UCB, Consultant of: AbbVie, Bristol-Myers Squibb, Celgene, Eli Lilly and Company, Gilead, Janssen, Novartis, Pfizer, and UCB, Stefan Siebert Grant/research support from: BMS, Boehringer Ingelheim, Celgene, GlaxoSmithKline, Janssen, Novartis, Pfizer, UCB, Consultant of: AbbVie, Boehringer Ingelheim, Janssen, Novartis, Pfizer, UCB, Speakers bureau: AbbVie, Celgene, Janssen, Novartis, Gerard Graham: None declared

DOI: 10.1136/annrheumdis-2020-eular.2980

\section{SAT0352 \\ AN UNSUPERVISED ANALYSIS IDENTIFIES A SPECIFIC IMPACT OF BIOLOGICS ONT LYMPHOCYTE PHENOTYPES.}

N. Rosine ${ }^{1}$, G. Millot ${ }^{2}$, S. Koturan ${ }^{3}$, C. Leloup ${ }^{3}$, H. Yahia ${ }^{3}$, E. Bianchi ${ }^{3}$, C. Miceli Richard $^{4,5}$, L. Rogge ${ }^{3,4} .{ }^{1}$ Pasteur Institute, Immunoregulation Unit, PARIS, France; ${ }^{2}$ Pasteur Institute, Bioinformatics and Biostatistics Hub, C3BI, PARIS, France; ${ }^{3}$ Pasteur Institute, Immunoregulation Unit, PARIS, France; ${ }^{4}$ Unité Mixte de Recherche, Institut Pasteur/AP-HP Hôpital Cochin, PARIS, France; ${ }^{5}$ Université Paris Descartes, Service de Rhumatologie B - Hôpital Cochin Assistance Publique - Hôpitaux de Paris, EULAR center of excellence, PARIS, France

Background: It is currently not known if TNF or IL-17A inhibitors have an impact on immune cell frequencies in axial Spondyloarthritis (AxSpA). This question is important to understand the impact of biologics on the immune system. Data from clinical trials didn't show significant modification on immune cells and especially on lymphocytes. But regarding the risk of infections linked to these treatments lymphocyte cell subsets are certainly disturbed. Moreover, biologics could affect subsets of cells with an unusual phenotype.

Objectives: To identify the phenotype of cell subsets affected by biologics.

Methods: We used an "unsupervised approach" to analyze CD4+ T cells and CD8+T cells subsets. Contrary to a "supervised approach", this strategy takes advantages of the fluorescence emitted by all of the surface markers used to characterize the cells at the same time. The objective was on the one hand to overcome statistical problems related to the number of patients and the repetition of the tests and on the other hand to increase the sensitivity of the analysis by identifying and analyzing new cell populations. The first step was to cluster the cells based on a selection of $12 \mathrm{~T}$ cells markers characteristic of the classica cell subsets and the stage of maturation to obtain cell clusters with a phenotype based on the combination of these 12 markers. Then, we were able to describe "a posteriori" the change of frequency of the clusters identified. The second step was to create a visualization of the cells affected to confirm their existence in a classical flow cytometry gate. With this pipeline, we analyzed CD4 and CD8 T cells isolated from a group of AxSpA patients $(n=7)$ before and after 3 months of TNF therapy and a group of patients $(n=6)$ before and after 4 months of IL-17A therapy.

Results: We observed that after biologics CD4 and CD8 T cells frequencies did not change but there was a redistribution of the different clusters analyzed. Specifically, we identified for CD4+T cells after anti TNF treatment an increase of 2 clusters (CD4+CD27+CD45RA+Va7.2intCD161int and CD4+CD27-CD45RA-CCR6+CD161int) and a decrease of 3 clusters (CD 4+CD27+CD45RA+CRTH2intCD161int, CD4+ CD27+CD45RA+CXCR3+, CD4+CD27+CD45RA+gdint CD161int) and for CD8+T cells a decrease of 1 cluster after treatment (CD8+CD27+CD45RA+CD161+CXCR3+) and an increase of 1 cluster (CD8+CD27+CD45RA+). The clusters affected by anti$\mathrm{IL}-17 \mathrm{~A}$ therapy were different. For CD4+T cells, we identified a decrease of 2 clusters (CD4+CD27+CD45RA+CXCR5+ CD161+ and CD4+CD27+CD45RACXCR3+CCR6+CD161+) and an increase of 2 clusters (CD4+CD27+CD45RA+gdintCD161+, CD4+CD27+CRTH2intCCR6+) and for CD8+T cells a decrease of 1 cluster (CD8+CD27+CD45RA+CXCR3+CRTH2intCD161int) and an increase of 1 cluster (CD8+CD27+CD45RA+CXCR3intCD161-).

Conclusion: We identified 5 different clusters in CD4+T cells affected by anti TNF and 4 by anti-IL-17A. We identified 2 clusters in CD8+T cells affected by anti TNF and 2 by anti-IL-17A. The phenotypes of these clusters were unexpected and raised new questions about the effect of biologics in AxSpA. We were also able to create a visualization of these cells affected by biologics in a "classic gating view" which will help us to perform scRNAseq. With this unique approach, we show an impact of biologics on the frequency of very specific subset of CD4+ and CD8+ T cells in AxSpA

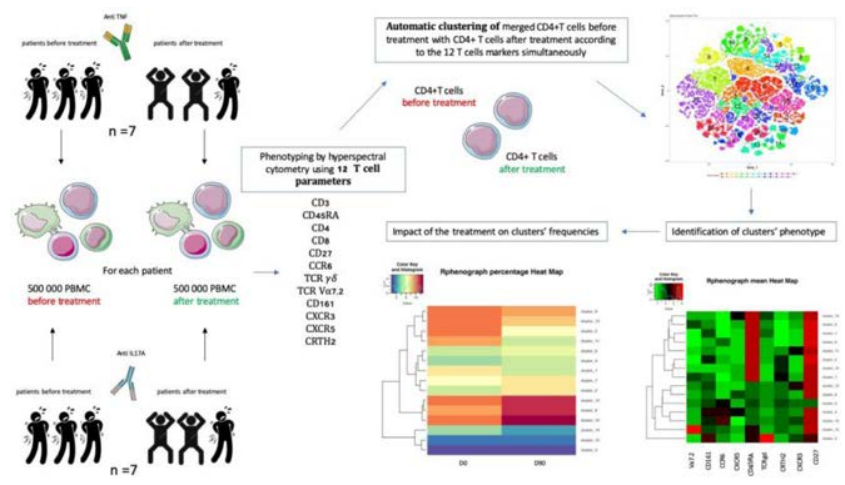

Figure 1.

Disclosure of Interests: None declared

DOI: 10.1136/annrheumdis-2020-eular.3838

SAT0353

STAT3 PHOSPHORYLATION IS INVOLVED IN THE DEVELOPMENTS OF INFLAMMATORY ARTHRITIS, ENTHESITIS, AND NEW BONE FORMATION IN ANKYLOSING SPONDYLITIS

S. H. Jin ${ }^{1}$, P. R. Park' ${ }^{1}$, M. J. Kim², Y. J. Lee ${ }^{2}$, S. Jo ${ }^{3}$, T. H. Kim³ ${ }^{3}$ J. Y. Kim ${ }^{4}$ S. C. Shim ${ }^{4}$, E. J. Won ${ }^{2}$, T. Kim ${ }^{1} .{ }^{1}$ Chonnam National University Medical School and Hospital, Rheumatology, Gwangju, Korea, Rep. of (South Korea); ${ }^{2}$ Chonnam National University Medical School, Gwangju, Korea, Rep. of 